\title{
Remote Monitoring of Patient Respiration with Mask Attachment-A Pragmatic Solution for Medical Facilities
}

\author{
Vivian Ci Ai Koh ${ }^{1, *(\mathbb{C}, \text {, Yi Yang Ang }}{ }^{1}\left(\mathbb{D}\right.$, Wee Ser ${ }^{1,2}$ and Rex Xiao Tan ${ }^{1}(\mathbb{D}$ \\ 1 Research and Development Department, Aevice Health Pte Ltd., 18 Howard Rd, \#06-11 Novelty BizCenter, \\ Singapore 369585, Singapore; yiyang@aevice.com (Y.Y.A.); ewser@ntu.edu.sg (W.S.); rex@aevice.com (R.X.T.) \\ 2 School of Electrical and Electronic Engineering, Nanyang Technological University, 50 Nanyang Ave, \\ Singapore 639798, Singapore \\ * Correspondence: vivian@aevice.com
}

Citation: Koh, V.C.A.; Ang, Y.Y.; Ser, W.; Tan, R.X. Remote Monitoring of Patient Respiration with Mask Attachment-A Pragmatic Solution for Medical Facilities. Inventions 2021, 6, 81. https://doi.org/10.3390/ inventions6040081

Academic Editor: Konstantinos G. Arvanitis

Received: 23 September 2021

Accepted: 29 October 2021

Published: 3 November 2021

Publisher's Note: MDPI stays neutral with regard to jurisdictional claims in published maps and institutional affiliations.

Copyright: (c) 2021 by the authors. Licensee MDPI, Basel, Switzerland. This article is an open access article distributed under the terms and conditions of the Creative Commons Attribution (CC BY) license (https:// creativecommons.org/licenses/by/ $4.0 /)$.

\begin{abstract}
Remote monitoring of vital signs in infectious patients minimizes the risks of viral transmissions to healthcare professionals. Donning face masks could reduce the risk of viral transmissions and is currently practiced in medical facilities. An acoustic-sensing device was attached to face masks to assist medical facilities in remotely monitoring patients' respiration rate and wheeze occurrence. Usability and functionality studies of the modified face mask were evaluated on 16 healthy participants. Participants were blindfolded throughout the data collection process. Respiratory rates of the participants were evaluated for one minute. The wheeze detection algorithm was assessed by playing 176 wheezes and 176 normal breaths through a foam mannequin. No discomfort was reported from the participants who used the modified mask. The mean error of respiratory rate was found to be $2.0 \pm 1.3$ breath per minute. The overall accuracy of the wheeze detection algorithm was $91.9 \%$. The microphone sensor that was first designed to be chest-worn has been proven versatile to be adopted as a mask attachment. The current findings support and suggest the use of the proposed mask attachment in medical facilities. This application can be especially helpful in managing a sudden influx of patients in the face of a pandemic.
\end{abstract}

Keywords: acoustic sensor; face mask; respiratory rate; wheeze

\section{Introduction}

At the time of writing this article, the world is combating a pandemic, Coronavirus Disease 2019 (COVID-19). New medical sub-industries such as telehealth have been widely and rapidly adopted as mainstream solutions [1,2]. Many technologies have extended the applications' original intentions to help elevate the current situation, such as adopting wearable devices and mobile applications for patient tracking and remote clinics [3].

Donning face masks has also become a new norm, both in and out of healthcare facilities. The World Health Organization (WHO) recommends individuals to wear a face mask in all public places as a mid-term measure in combating the spread of COVID-19 [4]. Consequently, face masks of different varieties came under the spotlight of researchers, businesses, and consumers alike, scrutinizing their effectiveness in protecting the wearer from the virus or preventing the wearer from becoming a source of infection if, unfortunately, infected [5,6].

Recent evidence suggests that face masks are integrals in deterring the spread of COVID-19 [7-9] when high compliance among the population is achieved [3,10,11]. In this context, Pan et al. proposed embedding a series of electronic sensors into a face mask to monitor blood oxygen saturation, heart rate, and temperature [12]. These vital physiological parameters are helpful to clinicians, especially when dealing with patients suffering from respiratory diseases. In line with these efforts, this article proposed a sensor device suitable for attaching on face masks, which listens to the wearer's breath, continuously monitors respiratory rate (RR), and detects any wheeze occurrence (WZ). 
Detection of a high RR range (>normal range) or WZ occurrence can indicate respiratory diseases such as COVID-19 pneumonia, asthma, and COPD exacerbation.

This work extends the recently proposed chest-worn wearable that addresses chronic respiratory disease care [13] and illustrates the novel applicability of repurposing the system as a mass patient-management and respiratory monitoring aid. The chest-worn wearable device was modified and repurposed for this work and is known in this article as the "mask attachment". To the authors' knowledge, this is the first report of integrating an acoustic-sensing mask attachment to medical face masks for mass patient monitoring. The contribution of the proposed system is not only limited to the efforts used in the combat of COVID-19, but it is also applicable for use in other teams' epidemic and pandemic threats that affect the respiratory system, such as the severe acute respiratory syndrome (SARS), Middle East Respiratory Syndrome (MERS), and Influenza A (H1N1). Furthermore, the proposed system, when compared to other noninvasive remote monitoring of RR methods such as the infrared (IR) [14] and near-infrared (NIR) [15] thermal sensing, can detect respiratory anomalies such as wheeze on top of RR monitoring. This proposed system also does not suffer from shortcomings of the aforementioned alternatives during mask wear, where important motion and heat signature from the face are covered.

\section{Materials and Methods}

\subsection{Sensing Device}

The sensing device is a microphone sensor coupled with an air conduction cavity formed by and enclosed with an Acrylonitrile Butadiene Styrene (ABS) thermoplastic enclosure. It weighs only $8 \mathrm{~g}$ with a cylindrical form of $10 \mathrm{~mm}$ in height and $31 \mathrm{~mm}$ in diameter. The collected breath sound from the sensor was transmitted to a docking station via a Bluetooth low energy (BLE) module for the computation of RR and WZ detection algorithms. The sampling rate of the microphone sensor is $4000 \mathrm{~Hz}$. The details of the sensor design and architecture are available in the references $[13,16,17]$.

\subsection{Experimental Setup}

KN95 surgical respirators (also known as the face mask in the study) were modified by substituting the round plastic enclosed air valve with a mask attachment, as shown in Figure 1. Sixteen participants were recruited for the study. Each participant's breath sound was recorded for one minute in an office environment, simulated as a clinic subjected to a common ambient noise level (averaged $50.5 \mathrm{~dB}$ over $1 \mathrm{~min}$ ). Participants were requested to breathe normally throughout the data collection.

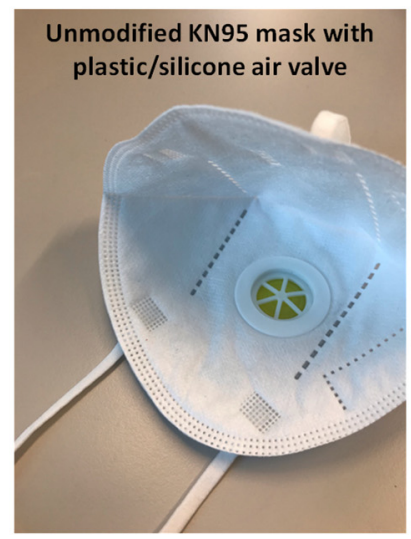

(a)

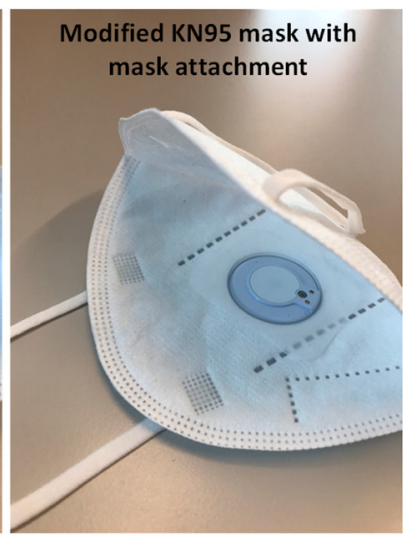

(b)

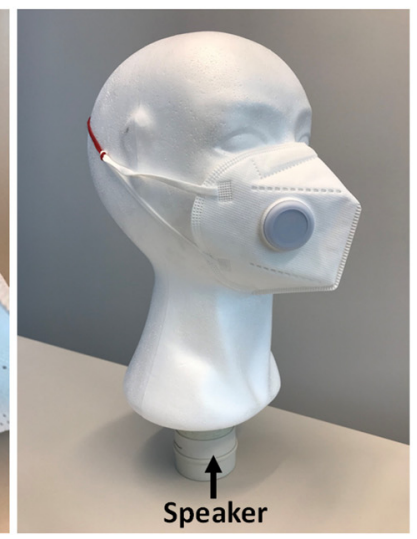

(c)

Figure 1. Experimental setup for both the user test and simulated wheeze and normal breath signal through the mannequin for the wheeze detection test. (a) The unmodified KN95 mask with a plastic/silicone air valve. (b) The modified KN95 mask with a miniature sensor inserted through a silicon patch. (c) A speaker was inserted at the bottom of the mannequin to mimic the travel of lung sound through trachea to the face level. 
All participants underwent the study blindfolded, with the procedure described in Figure 2. None experienced any respiratory complications during data collection.

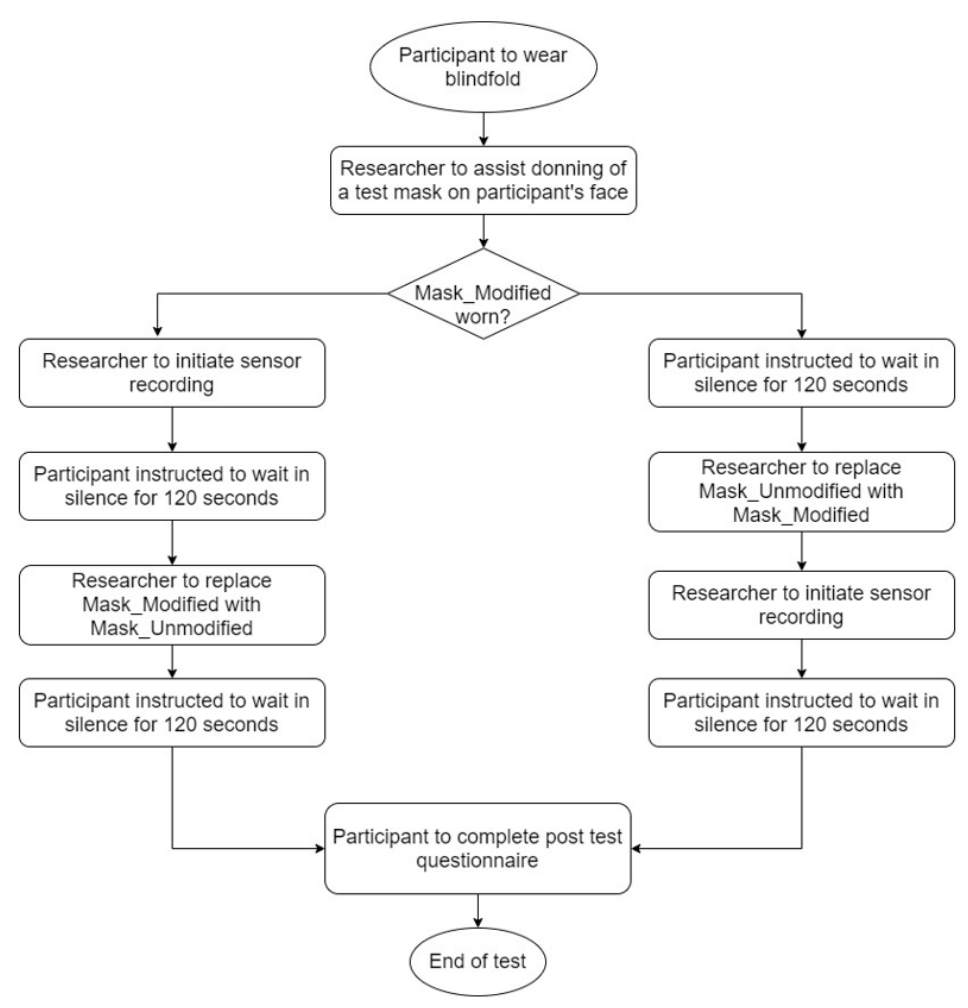

Figure 2. Usability test procedure.

\subsection{Human Factor Considerations}

For the proposed method to be readily accepted by the intended users, implementation of the technology should not hinder the user's range of motion or affect normal activities. Hence, the mask attachment must be minimally intrusive to encourage compliance. Since the wearer of the attachment would likely already be required to be wearing a facemask, this segment of the study focuses on the resulting implication of wearing the attachment on top of a common facemask. The translation of a series of human factor considerations in three categories of use environment, user and use interface, into a desired outcome and iteration of the process is well described by the United States Food Drug Administration's Guidance document [18].

\subsection{Use Environment}

The mask attachment is proposed for the application of mass patient management. The environment can then be either in a controlled indoor or outdoor medical facility where a wireless internet connection is available. The temperature and humidity of the environment are directly related to the comfort of mask wear [19]. Additionally, the mask attachment should not significantly lower breathability or promote additional condensation formation within the mask. The inclusion of an additional attachment on a facemask should also not impede communication capability through speech. Therefore, the mask attachment must only alter a small area of the facemask.

\subsection{User}

Medical grade facemasks, such as KN95 or N95 masks, come in various sizes and shapes to cater to the variant of possible face shapes and sizes. Sizing of these masks ensures proper fitment to achieve the intended outcome of air filtration for the wearer. Therefore, any attachment designed to be used with any mask should be physically small to fit facemasks' variation. 


\subsection{Use Interface}

The comfort, safety, and ease of use of the mask attachment are the main concern when designing the use interface. The modified mask weighs $14 \mathrm{~g}$, while the unmodified mask weighs around $8 \mathrm{~g}$ unaltered and is generally accepted by wearers. However, with the addition of an attachment, the increase in the mask's weight can impact the comfort of wear. In addition, the physical characteristics of the attachment must not result in significant impedance of respiration for the wearer by obstruction of airflow.

\subsection{Participants}

The 16 recruited participant profiles are summarized in Table 1. All participants have been wearing a face mask regularly for at least 6 months due to the COVID-19 pandemic.

Table 1. Participant profiles.

\begin{tabular}{cc} 
Attributes & Population \\
& $\boldsymbol{n}=\mathbf{1 6}$ \\
\hline Age (average) & $24.5 \pm 4.0$ \\
Gender & 5 \\
Male & 11 \\
Female & \\
Mask Size * & 1 \\
S (<100 mm) & 11 \\
M (100-110 mm) & 4 \\
L (110-125 mm) & 1 \\
Pre-existing chronic respiratory conditions &
\end{tabular}

\subsection{Respiratory Rate Estimation and Wheeze Detection Algorithms}

The RR estimation algorithm evaluates a 15-s breath segment. Next, a short-time Fourier-transform (STFT) was performed on the incoming signals, using a Hanning window size of $128 \mathrm{~ms}$ with a 50\% overlap size. The frequency bandwidth used for the calculation of Shannon's entropy was between $200 \mathrm{~Hz}$ and $1000 \mathrm{~Hz}$. Lastly, autocorrelation was performed on the entropy signal to estimate the RR. More details of the RR estimation algorithms are available in reference [13].

The WZ algorithm evaluates only two-and-a-half-second of breath segment. The entropy was calculated using a smaller Hanning window size of $32 \mathrm{~ms}$ with a $90 \%$ overlap size. The frequency bandwidth used for the calculation of Shannon's entropy was between $100 \mathrm{~Hz}$ and $2000 \mathrm{~Hz}$. Three features were extracted from the entropy data: (1) time-based spectral entropy difference (maximum-minimum), (2) time-based spectral entropy ratio (maximum/minimum), and (3) frequency-based spectral entropy difference (maximumminimum). The difference between the frequency-based and time-based spectral entropy features is that the entropy function was calculated in each row vector (frequency-based). Column vector (time-based) and the statistics (i.e., entropy difference and entropy ratio) were calculated across the row vectors (frequency-based) and column vectors (time-based) of the STFT outputs.

For no wheeze patient was recruited, a simulation using a speaker to play 176 random sample tracheal wheezes and 176 random sample normal breaths from an online database [20], through a foam mannequin, as shown in Figure 1, was employed to evaluate the WZ detection method. The simulation was repeated three times to account for varying environmental noise; thus, there were a total of 528 wheeze samples and 528 normal breath samples included in the data analysis. The mask attachment was worn on the mannequin to collect wheezes and normal breaths, as shown in Figure 1.

\subsection{Validation of Respiratory Rate Estimation and Wheeze Detection Algorithms}

The RRs calculated by the system were benchmarked with manual counts of breath periods within the 15-s evaluation windows. The manual counting method is the common 
practice nurses use to obtain the RR of their patients [21-23]. In the manual counting procedure, the start of each breath was annotated with a start time so that the duration of each breath cycle was derived as the interval between two consecutive start times. In our study, the reference RRs were first cross-validated by visual and audio inspection to determine each breath's start and end time within the one-minute recordings. The mean reference RRs were then computed for the one-minute recordings by that annotator. The reference RRs were further cross-validated by two medical students through audio inspection on the same one-minute recordings. Lastly, the final reference RRs used in the discussion of the results of this work were obtained by averaging all the annotations aforementioned for each recording of breathing sound. To account for the robustness of the wheeze detection classification method toward inter-subject variability, only $75 \%$ of the wheeze and normal breath samples were included in the acquisition of the SVM model. A total of $75 \%$ of the data samples were subdivided into $70 \%$ of training samples and $30 \%$ of testing samples. The performance of the SVM model was cross-validated using a 10-fold cross-validation method, and the final model was acquired. To further validate the capability of the wheeze detection algorithm on new data, the remaining $25 \%$ of data that had not been used in the acquisition of the SVM model were included in the final evaluation of model performance. The sensitivity and specificity of the WZ detection algorithm were calculated from the percentage of true positive (wheeze) detected and the percentage of true negative (normal breath) detected, respectively. The dataset used for the $\mathrm{WZ}$ detection test was pre-annotated for the presence and absence of wheeze by the database [20].

\section{Results and Discussion}

\subsection{Usability}

The success of a human-centered product design for any functional mask attachment can loosely be represented by the inability of the wearer to differentiate between wearing a mask with and without the attachment or experiencing any hindrance in human performance. As a qualitative analysis, 16 participants were asked to describe their experience according to the study design described in the earlier section, which involved deprived visual-sensory interaction of the participants with two masks. One of the two was modified with the mask attachment.

Ten out of 16 participants felt that the two masks were different. One participant expressed that the "motion of the sensor" was felt for the modified mask, and another reported that the sensor could be felt on the unmodified mask. As the mask attachment is a non-moving part and the unmodified mask does not contain the mask attachment, these two participants' experiences were inconsistent with the facts. Three participants reported the unmodified mask was more breathable, while two others reported the opposite. One of the two who reported the opposite said it may be due to the "tightness of the mask" worn on the unmodified mask. However, the tightness was not mentioned before the data collection session.

Five participants correctly reported the modified mask was slightly heavier than the unmodified mask. Nevertheless, no participant reported any significant discomfort experienced during or after the study.

\subsection{Respiratory Rate Estimation}

The normal range of resting RR for adults is 12-20 breath per minute (brpm) [24]. Table 2 shows that the resting RRs of 12 recruited participants were within the normal range, while the resting RRs of three others were above normal. 
Table 2. Comparison of resting respiratory rate between the mask attachment and manual counting for spontaneous breathing.

\begin{tabular}{cccc}
\hline \multirow{2}{*}{ Participant } & \multicolumn{2}{c}{ Respiratory Rate (brpm) } & Mean Absolute Error (brpm) \\
\cline { 2 - 3 } & Manual Count & Mask Attachment & \\
\hline 1 & 17.2 & 16.2 & 1.0 \\
2 & 18.9 & 22.0 & 3.1 \\
3 & NA & NA & NA \\
4 & 17.2 & 17.8 & 0.6 \\
5 & 12.5 & 16.5 & 4.0 \\
6 & 18.8 & 16.2 & 2.6 \\
7 & 13.9 & 17.6 & 3.7 \\
8 & 21.2 & 20.8 & 0.4 \\
9 & 23.1 & 19.8 & 3.3 \\
10 & 13.6 & 15.6 & 2.0 \\
11 & 20.0 & 19.0 & 1.0 \\
12 & 18.1 & 18.6 & 0.5 \\
13 & 18.9 & 15.8 & 3.1 \\
14 & 21.7 & 20.8 & 0.9 \\
15 & 20.0 & 20.7 & 0.7 \\
16 & 19.8 & 23.0 & 3.2 \\
\hline
\end{tabular}

NA: Not available.

The algorithm could accurately measure RR in all ranges, including the range that is outside of normal range (i.e., $>20 \mathrm{brpm}$ ). The RR estimation algorithm's smallest and largest mean absolute errors were $0.2 \mathrm{brpm}$ and $4.0 \mathrm{brpm}$, respectively. Autocorrelation function has also been proven useful in extracting the periodicity of quasi-periodic physiological signals [25]. However, the accuracy of such a method decreases with increasing interperiod variability of RR. The results showed that the RR algorithm achieved an overall high accuracy (with a mean absolute error of $2.0 \pm 1.3 \mathrm{brpm}$ ) among the 15 participants. The reference of participant 3 was not available as the breath sound recording was not heard by the annotators. Thus, the results of participant 3 were excluded from the study.

The main challenge of the RR estimation algorithm was to accurately identify the inspiration and expiration of a breath and calculate the number of repeated patterns within a 15-s window, especially in cases where the duration of inspiration and expiration is equal.

Figure 3 demonstrates the risk of overestimating and underestimating the RR. Figure 3a,c showed that the actual RR was $12.9 \mathrm{brpm}$, but the RR algorithm was estimated as $18.4 \mathrm{brpm}$. Figure $3 \mathrm{~b}$ shows a burst split in the exhale between $10.5 \mathrm{~s}$ and $12.5 \mathrm{~s}$ within the same breath cycle. This may have contributed to the overestimation of the RR algorithm as it considered this short period of breath cycle in the evaluation window. Conversely, Figure 3d,f demonstrate the risk of underestimating the RR. The actual RR was $15.1 \mathrm{brpm}$, but the RR algorithm estimated $7.1 \mathrm{brpm}$. Such error occurred because the breath patterns were irregular (higher variability in the duration of inspiration and expiration and period of breath cycles within the evaluation window), and that was challenging for the algorithm to detect the global peaks in the autocorrelation plot in Figure 3f.

Even though the gold standard of respiratory rate monitoring is capnometry, the most common method while the most common method used in the clinic is manual counting by healthcare professionals [26,27]. Nevertheless, manual counting of RR is labor-intensive and unsuitable for continuous or prolonged RR measurement, especially for healthcare facilities that had been overwhelmed.

Still, manual counting of a one-minute sample recording is widely practiced in various evaluation protocols [28-30]. For this reason, only a one-minute sample was recorded for each subject for the evaluation of the RR algorithm. As the RR algorithm computes a 15-s segment data, with an update rate of 5-s, there will be 10 RR outputs in every one-minute recording. This is sufficient to validate the RR algorithm to prove a new application concept. 


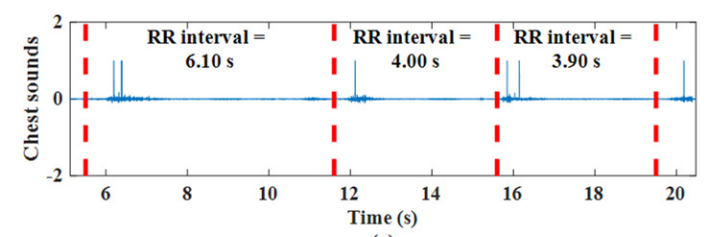

(a)

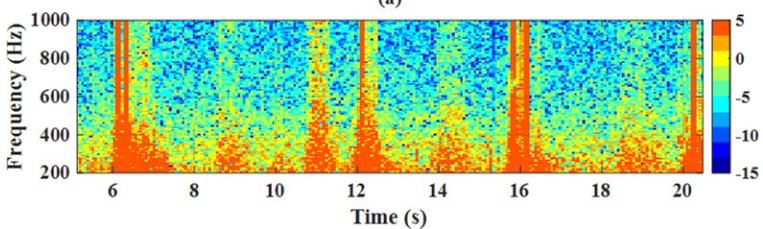

(b)

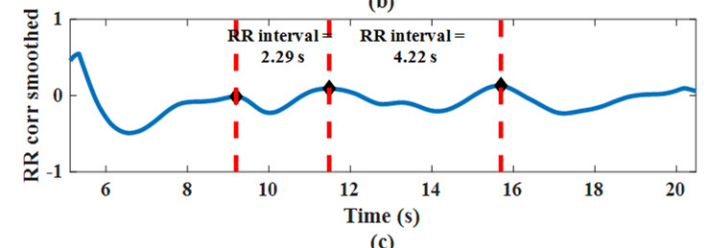

(c)

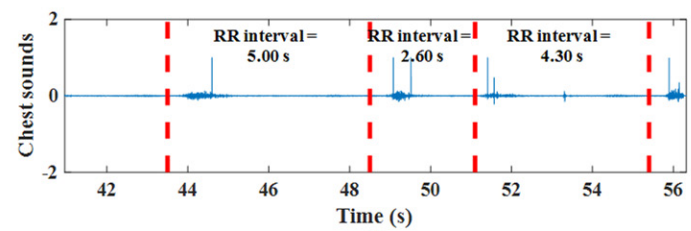

(d)
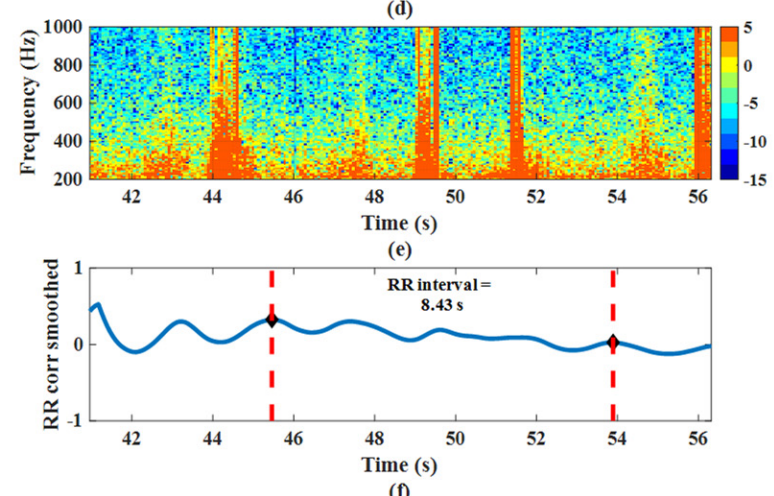

(f)

Figure 3. A short segment plot of (a-c) participant 10's recording (estimated RR > actual RR) and (d-f) participant 10's recording (estimated RR < actual RR) in the time-domain between $200 \mathrm{~Hz}$ and $1000 \mathrm{~Hz}$, time-domain entropy plot of breath sounds, and autocorrelation plot of entropy signals.

\subsection{Wheeze Detection}

The WZ detection algorithm was originally designed to work on chest sounds, using two low-complexity time-based spectral entropy features to differentiate wheezes from normal breaths. In the present study, another feature, frequency-based entropy difference, was added to the WZ detection algorithm to improve classification accuracy. As listed in Table 3, the sensitivity, specificity, and accuracy of the simulated tracheal normal breaths and wheeze classified using a support vector machine (SVM) model with a radial basis kernel function were $88.8 \%, 94.9 \%$, and $91.9 \%$, respectively. Thus, the wheeze detection algorithm shows a balanced trade-off between sensitivity and specificity, which is an important criterion for a "good classifier". In addition, the model performance was evaluated using a 10-fold cross-validation method to show that the model was robust when tested in 10 different scenarios.

Table 3. Performance of wheeze detection algorithm.

\begin{tabular}{cccc}
\hline \multirow{2}{*}{ Actual Breath Classification } & \multicolumn{2}{c}{ Classification by Wheeze Detection Algorithm } & \\
\cline { 2 - 4 } & Wheeze & Normal Breath & Sensitivity: $88.8 \%$ \\
\hline Wheeze & 469 & 59 & Specificity: $94.9 \%$ \\
\hline Normal breath & 27 & 501 & Accuracy: $91.9 \%$ \\
\hline
\end{tabular}

Even though a mannequin with a speaker does not represent an actual wheezy patient, it is a reasonable simulation that shows the direction of sound from the chest level through the airway to the sensor attached to the mask for a proof-of-concept study as simulations have been used in other studies [31,32]. In future studies, the wheeze detection algorithm will be validated in a clinical trial with actual wheezing patients.

The current study shows that the acoustic sensor is versatile for a different application, i.e., a mask attachment. Functionally, the original intended use of the sensor at the chest level provides a more comprehensive cardiopulmonary monitoring as it also estimates heart rate together with RR. However, the current proposed application provides an easier mode of administration and a more comfortable solution (with no direct surface contact of the sensor with the patient) to remotely monitor RR and detect wheeze. Notably, RR 
has also been recognized as the first indication and the best marker of deterioration in a patient's conditions [33]. Therefore, the results from the study support that the proposed mask attachment can help manage patient conditions in overwhelmed clinics.

\section{Conclusions}

Encountering the pandemic of COVID-19, donning a face mask becomes essential and even compulsory in certain countries as part of the effort in curtailing the spread of the virus among the community. The mask attachment was proposed for mass patient monitoring. The comfort of using the modified face mask has been evaluated through the verdicts of 16 participants in the usability study. All participants reported no discomfort using the modified masks, although about half felt differences between the unmodified and modified masks. Lastly, the overall performance of RR estimation and WZ detection algorithms have shown that the sensor system, originally developed for chest-worn sensing, can be versatilely adopted into this work as mask attachment. Future validation of the application in a real clinical scenario in a larger sample size may be warranted to increase confidence, acceptability, and favor among the practitioners in clinics.

\section{Patent}

A significant portion of the technology presented in this work is patent pending in Singapore with the application number 10202004626V filed by Aevice Health Pte Ltd., 18 Howard Rd, \#06-11 Novelty BizCenter, Singapore 369585.

Author Contributions: V.C.A.K., Y.Y.A. and R.X.T. conceptualized, designed, and conducted the study. All authors analyzed and discussed the results. V.C.A.K. and Y.Y.A. prepared the manuscript. R.X.T. and W.S. reviewed and revised the manuscript. All authors have read and agreed to the published version of the manuscript.

Funding: This research is funded by Aevice Health Pte Ltd., a medical device research and development company based in Singapore.

Institutional Review Board Statement: Ethical review and approval were waived for this study. All methods were carried out in accordance with the Human Biomedical Research Act of Singapore 2015, revised in 2019. With reference to part 1, Section 3.2 of the Human Biomedical Research Act of Singapore, part of this study where human participants were involved is not intended to investigate the prevention, prognostication, diagnosis or alleviation of any disease, disorder or injury affecting the human body; the restoration, maintenance, or promotion of the aesthetic appearance of human individuals through clinical procedures or techniques; or the performance or endurance of human individuals. Only healthy participants were recruited, and information collected were simple recordings of their breathe sound. Therefore, an approval of the protocol from an institutional board or licensing is not deemed to be required. All participants are employees of Aevice Health Pte Ltd, Singapore.

Informed Consent Statement: An informed consent was obtained from all participants involved in the study.

Data Availability Statement: The data presented in this study are available on request from the corresponding author. The data are not publicly available due to privacy restrictions.

Conflicts of Interest: The authors declare no conflict of interest. Rex Xiao Tan and Wee Ser are the founders of Aevice Health Pte Ltd., in which they hold shares. Vivian Ci Ai Koh, Yi Yang Ang, and Rex Xiao Tan are employees of Aevice Health Pte Ltd. The company had no role in the design, collection, analyses, or interpretation of data, the writing of the manuscript, or the decision to publish the results.

\section{References}

1. Portnoy, J.; Waller, M.; Elliott, T. Telemedicine in the era of COVID-19. J. Allergy Clin. Immunol. Pract. 2020, 8, 1489-1491. [CrossRef]

2. Mann, D.M.; Chen, J.; Chunara, R.; Testa, P.A.; Nov, O. COVID-19 transforms health care through telemedicine: Evidence from the field. J. Am. Med. Inform. Assoc. 2020, 27, 1132-1135. [CrossRef] 
3. Levine, D.M.; Ouchi, K.; Blanchfield, B.; Diamond, K.; Licurse, A.; Pu, C.T.; Schnipper, J.L. Hospital-level care at home for acutely ill adults: A randomized controlled trial. Ann. Intern. Med. 2020, 172, 77-85. [CrossRef] [PubMed]

4. World Health Organization. Advice on the Use of Masks in the Context of COVID-19: Interim Guidance, 6 April 2020; World Health Organization: Geneva, Switzerland, 2020.

5. Verma, S.; Dhanak, M.; Frankenfield, J. Visualizing the effectiveness of face masks in obstructing respiratory jets. Phys. Fluids 2020, 32, 61708. [CrossRef]

6. Chughtai, A.A.; Seale, H.; Macintyre, C.R. Effectiveness of cloth masks for protection against severe acute respiratory syndrome coronavirus 2. Emerg. Infect. Dis. 2020, 26, e200948. [CrossRef] [PubMed]

7. Eikenberry, S.E.; Mancuso, M.; Iboi, E.; Phan, T.; Eikenberry, K.; Kuang, Y.; Kostelich, E.; Gumel, A.B. To mask or not to mask: Modeling the potential for face mask use by the general public to curtail the COVID-19 pandemic. Infect. Dis. Model. 2020, 5, 293-308. [CrossRef]

8. Wang, J.; Pan, L.; Tang, S.; Ji, J.S.; Shi, X. Mask use during COVID-19: A risk adjusted strategy. Environ. Pollut. 2020, 266, 115099. [CrossRef] [PubMed]

9. Cheng, V.C.-C.; Wong, S.-C.; Chuang, V.W.-M.; So, S.Y.C.; Chen, J.H.K.; Sridhar, S.; To, K.K.W.; Chan, J.F.W.; Hung, I.F.N.; Ho, P.L.; et al. The role of community-wide wearing of face mask for control of coronavirus disease 2019 (COVID-19) epidemic due to SARS-CoV-2. J. Infect. 2020, 81, 107-114. [CrossRef]

10. Duc, H.T.L. The COVID-19 containment in Vietnam: What are we doing? J. Glob. Health 2020, 10, 010338.

11. Chiang, C.-H.; Chiang, C.-H.; Chiang, C.-H. Maintaining mask stockpiles in the COVID-19 pandemic: Taiwan as a learning model. Infect. Control. Hosp. Epidemiol. 2021, 42, 244-245. [CrossRef]

12. Pan, L.; Wang, C.; Jin, H.; Li, J.; Yang, L.; Zheng, Y.; Wen, Y.; Tan, B.H.; Loh, X.J.; Chen, X. Lab-on-Mask for Remote Respiratory Monitoring. ACS Mater. Lett. 2020, 2, 1178-1181. [CrossRef]

13. Koh, V.C.A.; Tan, R.X.; Ang, Y.Y. A Miniature Wearable Microphone Sensor for Cardiopulmonary Monitoring. In Proceedings of the 2020 IEEE Sensors, Rotterdam, The Netherlands, 25-28 October 2020; IEEE: Piscataway, NJ, USA, 2020; pp. 1-4.

14. Pereira, C.B.; Yu, X.; Czaplik, M.; Rossaint, R.; Blazek, V.; Leonhardt, S. Remote monitoring of breathing dynamics using infrared thermography. Biomed. Opt. Express 2015, 6, 4378-4394. [CrossRef] [PubMed]

15. Jakkaew, P.; Onoye, T. Non-contact respiration monitoring and body movements detection for sleep using thermal imaging. Sensors 2020, 20, 6307. [CrossRef] [PubMed]

16. Ser, W.; Zhang, J.; Yu, J.; Zhang, T. Air Conduction Sensor and a System and a Method for Monitoring a Health Condition. U.S. Patent 9,826,955, 28 November 2017.

17. Ibrahim, A.; Koh, V.C.A.; Hamidah, S.; Ang, Y.Y.; Tan, R.X.; Ser, W. System and Method for Monitoring a Pluraity of Bio-Signals, and a Gateway Device Operable Therein. 17 May 2021.

18. Center for Devices \& Radiological Health. Human Factors and Usability Engineering to Medical Devices. 2019. Available online: https:/ / www.fda.gov/regulatory-information/search-fda-guidance-documents/applying-human-factors-and-usabilityengineering-medical-devices (accessed on 1 September 2020).

19. Scheid, J.L.; Lupien, S.P.; Ford, G.S.; West, S.L. Commentary: Physiological and psychological impact of face mask usage during the COVID-19 pandemic. Int. J. Environ. Res. Public Health 2020, 17, 6655. [CrossRef] [PubMed]

20. Rocha, B.M.; Filos, D.; Mendes, L.; Serbes, G.; Ulukaya, S.; Kahya, Y.P.; Jakovljevic, N.; Turukalo, T.L.; Vogiatzis, I.M.; Perantoni, E.; et al. An open access database for the evaluation of respiratory sound classification algorithms. Physiol. Meas. 2019, $40,35001$. [CrossRef] [PubMed]

21. Chan, P.; Wong, G.; Nguyen, T.D.; Hopper, I. Estimation of respiratory rate using infrared video in an inpatient population: An observational study. J. Clin. Monit. Comput. 2020, 34, 1275-1284. [CrossRef]

22. Jarchi, D.; Rodgers, S.J.; Tarassenko, L.; Clifton, D.A. Accelerometry-Based Estimation of Respiratory Rate for Post-Intensive Care Patient Monitoring. IEEE Sens. J. 2018, 18, 4981-4989. [CrossRef]

23. L'Her, E.; Nazir, S.; Pateau, V.; Visvikis, D. Accuracy of noncontact surface imaging for tidal volume and respiratory rate measurements in the ICU. J. Clin. Monit. Comput. 2021, 1-9. [CrossRef]

24. McGinley, A.; Pearse, R.M. A national early warning score for acutely ill patients. BMJ 2012, 345, e5310. [CrossRef]

25. Muller, J.L.; Hohlfeld, R.G. System for Measuring the Period of a Quasi-Periodic Signal. U.S. Patent 5,584,295, 7 December 1996.

26. Subbe, C.P.; Kinsella, S. Continuous monitoring of respiratory rate in emergency admissions: Evaluation of the RespiraSense ${ }^{\mathrm{TM}}$ sensor in acute care compared to the industry standard and gold standard. Sensors 2018, 18, 2700. [CrossRef]

27. Philip, K.E.J.; Pack, E.; Cambiano, V.; Rollmann, H.; Weil, S.; O’Beirne, J. The accuracy of respiratory rate assessment by doctors in a London teaching hospital: A cross-sectional study. J. Clin. Monit. Comput. 2015, 29, 455-460. [CrossRef]

28. Erden, F.; Alkar, A.Z.; Cetin, A.E. Contact-free measurement of respiratory rate using infrared and vibration sensors. Infrared Phys. Technol. 2015, 73, 88-94. [CrossRef]

29. Trimpop, J.; Schenk, H.; Bieber, G.; Lämmel, F.; Burggraf, P. Smartwatch based respiratory rate and breathing pattern recognition in an end-consumer environment. In Proceedings of the 4th international Workshop on Sensor-based Activity Recognition and Interaction, Rostock, Germany, 21-22 September 2017; pp. 1-5.

30. Laurie, J.; Higgins, N.; Peynot, T.; Fawcett, L.; Roberts, J. An evaluation of a video magnification-based system for respiratory rate monitoring in an acute mental health setting. Int. J. Med. Inform. 2021, 148, 104378. [CrossRef] 
31. Brown, C.; Morse, J.; Nesvadba, D.; Meldrum, A. Twelve tips for introducing simulation based assessment in the objective structured clinical examination. Med. Teach. 2020, 43, 380-383. [CrossRef]

32. Pawlowski, J.; Feinstein, D.; Crandall, M.L.; Gala, S. Modernizing Biomedical Training: Replacing Live Animal Laboratories with Human Simulation. Anim. Exp. Work. Towards A Paradig. Chang. 2019, 551-566. [CrossRef]

33. Armitage, M.; Eddleston, J.; Stokes, T. Recognising and responding to acute illness in adults in hospital: Summary of NICE guidance. BMJ 2007, 335, 258-259. [CrossRef] 